\title{
Análisis de los eventos desfavorables como resultado de la atención en cirugía oral
}

\author{
Analysis of Unfavorable Events Resulting from the Oral Surgery Health Care
}

\author{
Juan Martín Pesántez Alvarado \\ Práctica privada, Cuenca, Ecuador \\ martinchopesantez@gmail.com
}

Julián Danilo Camacho Ladino

Práctica privada, Bogotá, Colombia

julkmacho_02@hotmail.com

Adriana Rodríguez Ciódaro

Pontificia Universidad Javeriana, Colombia

arodrig@javeriana.edu.co

\author{
Sandra Patricia Camacho Peña Pontificia \\ Universidad Javeriana, Colombia \\ sandra-camacho@javeriana.edu.co
}

Ana Lucia Sarralde Delgado

Pontificia Universidad Javeriana, Colombia

sarralde@javeriana.edu.co

Diego Ernesto Castro Haiek

Pontificia Universidad Javeriana, Colombia

castro.d@javeriana.edu.co

Juliana González Moncada

Pontificia Universidad Javeriana, Colombia

juliana.gonzalez@javeriana.edu.co

\section{Resumen:}

Antecedentes: La cirugía oral es una especialidad que requiere preparación teórica y habilidad quirúrgica para efectuar los procedimientos. Durante el proceso de aprendizaje, los estudiantes, al efectuar cirugías, pueden enfrentar dificultades que dañen involuntariamente al paciente. A pesar de cumplirse los protocolos, es posible que se presenten eventos desfavorables durante la atención. Por este motivo, es necesario caracterizar y analizar dichos eventos en instituciones como una facultad de odontología para mejorar la calidad de la atención. Objetivo: Analizar los eventos desfavorables que se presentaron en el área quirúrgica de la Facultad de Odontología de la Pontificia Universidad Javeriana en un periodo de dos años. Métodos: En este estudio observacional-descriptivo se evaluaron todas las historias clínicas de pacientes atendidos entre el 1 de enero de $2014 \mathrm{y}$ el 31 de diciembre de 2015, a fin de identificar y analizar los eventos desfavorables en cirugía oral. Resultados: De 1062 historias clínicas, 74 (7\%) tenían reportes de eventos desfavorables, 41 (56\%) de las cuales se tipificaron como complicaciones, 22 (30 \%) como indicios de atención insegura y $11(14 \%)$ como eventos adversos. De ellos, 9 (82 \%) fueron prevenibles y 2 (18 \%) no prevenibles, todos relacionados con exodoncias. Conclusiones: Los eventos adversos en el área quirúrgica se presentaron en baja frecuencia y se relacionaron con exodoncias. Para reducir la ocurrencia de estas situaciones, es preciso realizar planeación prequirúrgica, elaborar meticulosamente las historias clínicas y, en caso de presentarse algún tipo de evento, informarlo. Palabras clave: atención en salud, cirugía oral, complicación en cirugía oral, error en cirugía ora, evento adverso, evento desfavorable, seguridad del paciente.

Áreas temáticas: administración en salud; cirugía oral; odontología. 


\begin{abstract}
Background: Oral surgery is a specialty requiring both theoretical formation and training in surgical skills in order to carry out the procedures. During the learning process when students carry out surgeries, they may face difficulties that involuntarily harm the patient. Despite adhering to the protocols, it is possible that unfavorable events occur while providing dental care to the patients. Therefore, there is a need to characterize and analyze those events in institutions such as the dentistry schools in order to improve the health care quality. Objective: To analyze the unfavorable events that occurred in the surgery room of the Pontificia Universidad Javeriana Dentistry School during a two-year study term. Methods: In this observational-descriptive study all the medical records taken to patients who attended to the dentistry school from January 1, 2014 to December 31, 2015 were evaluated in order to i dentify and analyze the unfavorable events occurred during the oral surgeries. Results: Out of 1062 medical records, 74 (7\%) r eported unfavorable events and 41 (56\%) of them were characterized as complications, $22(30 \%)$ as a lack of self-confidence by the health care giver, and $11(14 \%)$ as adverse events. In addition, $9(82 \%)$ were preventable and 2 (18\%) non-preventable events, all of them related to dental extractions. Conclusions: Adverse events in the oral surgery area were infrequent and related mostly with dental extractions. To reduce their occurrences, it is important to do a preoperative planning, to prepare carefully the medical r ecords and, in the case of any occurrence, to report it.
\end{abstract}

Keywords: health care, oral surgery, oral surgery complications, error in oral surgery, adverse event, unfavorable event, patient safety.

Thematic fields: dentistry; health management; oral surgery

\title{
INTRODUCCIÓN
}

Entre las prácticas en salud, un evento adverso es el resultado de una atención que, de manera no intencional, daña al paciente. Dichos eventos se pueden clasificar como prevenibles y no prevenibles. Es importante diferenciar entre un evento adverso y una complicación, pues esta última se refiere a un daño o resultado clínico no esperado que no es atribuible a la atención en salud, sino a la enfermedad o a las condiciones propias del paciente $(1,2,3)$.

Se estima que, cada año, decenas de millones de pacientes en el mundo sufren lesiones incapacitantes o mueren como consecuencia de prácticas médicas o atención insegura. Por tal razón, en 1999 se definieron políticas mundiales encaminadas a garantizar la seguridad del paciente, que han contribuido a mejorar la atención en las entidades prestadoras de servicios de salud (4). En 2002, la Organización Mundial de la Salud, durante la Asamblea Mundial de la Salud celebrada en Ginebra, Suiza, aprobó la resolución WHA55.18, cuyo propósito es establecer sistemas necesarios para mejorar la seguridad del paciente y, por ende, la calidad en la atención en salud (4). Posteriormente, en octubre de 2004, la Organización Mundial de la Salud creó la Alianza Mundial para la Seguridad del Paciente, que se encarga de estructurar programas con aspectos sistémicos y técnicos en todo el mundo. En ese mismo año, se incluyó una serie de medidas, consideradas clave para reducir el número de enfermedades, traumatismos y defunciones que pudieran sufrir los pacientes al recibir atención sanitaria. Su objetivo puede resumirse en el lema: "Ante todo, no hacer daño" (4).

Uno de los objetivos de la Alianza es disminuir la duplicación de actividades e inversión de recursos, apoyar iniciativas colectivas, servir como vehículo para compartir conocimientos y recursos, "aprender de los demás" y promover el trabajo en equipo. A pesar de toda la voluntad por implantar medidas preventivas de seguridad para el paciente y disminuir así la ocurrencia de eventos adversos, estos se continúan presentando en todas las profesiones de la salud (4).

Por otra parte, se han propuesto mecanismos para notificar los eventos adversos; pero debido a factores de rechazo o temor a ser juzgado, el profesional tiende a omitir el informe. Por lo tanto, es indispensable que los profesionales comprendan que, al realizar tales reportes, facilitan la retroalimentación de las posibles fallas, incidentes, errores y complicaciones que se pueden dar durante una práctica clínica, como la única manera de instaurar medidas preventivas y correctivas (4).

En Latinoamérica, el primer estudio que reportó el problema de la seguridad fue el Estudio Iberoamericano de Efectos Adversos (IBEAS), realizado en 5 países de la región (México, Costa Rica, Colombia, Perú y Argentina). El IBEAS evidenció que, en promedio, uno de cada 10 pacientes hospitalizados $(10 \%)$ sufre al menos un daño durante la atención en estos países. Posteriormente, el 
Estudio de Prevalencia de Eventos Adversos en la Atención Ambulatoria, realizado en 4 países de Latinoamérica, se enfocó en la capacidad de los pacientes como informantes de eventos adversos graves. De 2080 pacientes entrevistados, la prevalencia de eventos adversos fue del $5 \%$ al $6 \%$ (5). En Colombia, el IBEAS mostró una prevalencia de eventos adversos del 13,1\%, 27,3\%, de los cuales se presentaron en menores de 15 años (5).

El Ministerio de Salud de Colombia, mediante la Resolución 1446 del 2006, estableció la obligatoriedad de todos los actores del Sistema Obligatorio de Garantía de Calidad, para notificar y vigilar la ocurrencia de eventos adversos. Uno de los propósitos del sistema de salud es mejorar la calidad de vida de las poblaciones, al brindar calidad en la atención en salud y, de esta manera, proporcionar una atención segura y ética sin ocasionar daño (6). Con el propósito de mejorar la seguridad del paciente en las instituciones y con la Resolución 2003 de 2014 como marco legal, se estableció la obligatoriedad de tener una estructura dedicada exclusivamente a la seguridad del paciente $y$, de esta manera, minimizar la frecuencia de eventos adversos en la sociedad colombiana $(5,6,7)$.

La Facultad de Odontología de la Pontificia Universidad Javeriana en Bogotá, Colombia, ofrece estudios de especializaciones en Patología y Cirugía Bucal, en la cual se practican procedimientos quirúrgicos que son realizados por los estudiantes y supervisados por sus profesores. Frente a la obligación de asegurar la calidad de atención de los pacientes y, con la consideración de ser una institución de formación de profesionales para la práctica clínica en salud, en la facultad se han realizado investigaciones sobre la naturaleza y frecuencia de los eventos adversos presentados en áreas como endodoncia (8) y periodoncia (9), así como el análisis de las quejas que se reportan ante la Dirección de Clínicas de la Facultad (10). En todos los casos, la frecuencia de presentación de los eventos adversos ha sido baja y, en su mayoría, de carácter prevenible. Este tema no se había abordado para los procedimientos que se realizan en el área quirúrgica, por lo cual se formuló la pregunta: ¿cuáles son la frecuencia y las características de las situaciones desfavorables que se presentan por la atención de pacientes en el área quirúrgica de la Facultad de Odontología de la Pontificia Universidad Javeriana de Bogotá, Colombia?

En el contexto de la cirugía oral existen distintos tipos de tratamientos que requieren el seguimiento de protocolos estrictos. A pesar de tenerlos incluidos, existe la posibilidad de que aparezcan situaciones desfavorables antes del procedimiento quirúrgico, durante este y después. Los más reportados en la literatura sobre el tema son: alveolitis (11); reacción adversa al anestésico o al vasoconstrictor (12,13); desplazamiento de dientes a espacios anatómicos durante la exodoncia (14); lesiones nerviosas (15); daño a estructuras adyacentes, dientes o seno maxilar e, incluso, la muerte del paciente (16); hemorragias (17); fractura de tejidos duros, y laceración a tejidos blandos (18).

Por lo anterior, el objetivo de este trabajo fue analizar los eventos desfavorables que se presentaron durante la atención de pacientes en el área quirúrgica de la Facultad de Odontología de la Pontificia Universidad Javeriana, entre 2014 y 2015.

\section{MATERIALES Y MÉTODOS}

Este estudio observacional-descriptivo recibió el aval del Comité de Investigación y Ética de la Facultad de Odontología de la Pontificia Universidad Javeriana (Acta 013 de 2012). Se decidió analizar todas las historias clínicas de los pacientes atendidos en las clínicas del posgrado de Patología y Cirugía Oral del 1 de enero de 2014 al 31 de diciembre de 2015, teniendo en cuenta la baja frecuencia de eventos adversos que se han encontrado en otras áreas estudiadas en la misma universidad.

En un instrumento de recolección de la información diseñado para tal fin se registraron las siguientes variables para cada una de las historias analizadas: edad, sexo, estado civil, tipo de aseguramiento en el sistema de salud colombiano, presencia o no de afectación sistémica y tipo de procedimiento quirúrgico realizado. 
Para las historias que incluían reportes de eventos desfavorables, se registró adicionalmente el tipo de evento presentado.

Con el objetivo de tipificar los eventos desfavorables presentados, se reunieron los expertos del área quirúrgica de la misma Facultad, en la que inicialmente se socializaron los conceptos emitidos por el Ministerio de Salud y Protección Social a través de sus guías de seguridad (5,6,7). Después se presentaron cada uno de los casos que, por consenso, se clasificaron como indicios de atención insegura, complicaciones o eventos adversos prevenibles o no prevenibles.

La información se tabuló y se estableció la frecuencia con que se presentaron y se analizaron las diferentes variables que podían haber influido en una situación desfavorable. Los datos se analizaron usando estadística descriptiva.

\section{RESULTADOS}

De las 1062 historias evaluadas, 637 correspondieron a mujeres (60\%) y, aproximadamente, la mitad de las personas tenía entre 16 y 35 años de edad. El procedimiento que más se realizó fue la exodoncia (tabla 1), especialmente en personas jóvenes, seguido de la colocación de implantes, que aumentó a partir de los 45 años (figura 1). El $83 \%$ de los pacientes no presentaba afectaciones sistémicas. La mayor parte de las personas pertenecía al régimen contributivo y el estado civil predominante fue soltero (tabla 1 ).

TABLA 1

Distribución de las variables registradas a partir de las historias clínicas analizadas

\begin{tabular}{llr}
\hline \multicolumn{2}{c}{ Nombre de la variable } & Número de casos (porcentaje) \\
\hline Tipos de & Exodoncia & $738(69,0)$ \\
procedimientos & Implante & $149(14,0)$ \\
& Segunda fase & $72(7,0)$ \\
& quirúrgica & \\
& Biopsias & $57(5,0)$ \\
& Ventanas quirúrgicas & $14(1,0)$ \\
& Cirugía preprotésica & $9(1,0)$ \\
& Frenillectomías & $6(0,5)$ \\
& Enucleación & $5(0,5)$ \\
& Elevación de seno & $6(0,5)$ \\
Grupos etarios & Injertos óseos & $6(0,5)$ \\
(en años) & $16-25$ & $63(5,0)$ \\
& $26-35$ & $451(42,0)$ \\
& $36-45$ & $152(14,0)$ \\
& $46-55$ & $67(6,0)$ \\
Tipo de & S5 & $99(9,0)$ \\
aseguramiento & Contributivo & $230(22,0)$ \\
& Vinculado & $253(24,0)$ \\
& Otro & $729(69,0)$ \\
& Ninguno & $44(4,0)$ \\
Estado civil & Soltero(a) & $17(1,0)$ \\
& Casado(a) & $19(2,0)$ \\
& Unión libre & $740(70,0)$ \\
& Viudo(a) & $198(19,0)$ \\
& Divorciado(a) & $63(6,0)$ \\
& & $31(2,0)$ \\
& & $40(3,0)$ \\
\hline
\end{tabular}




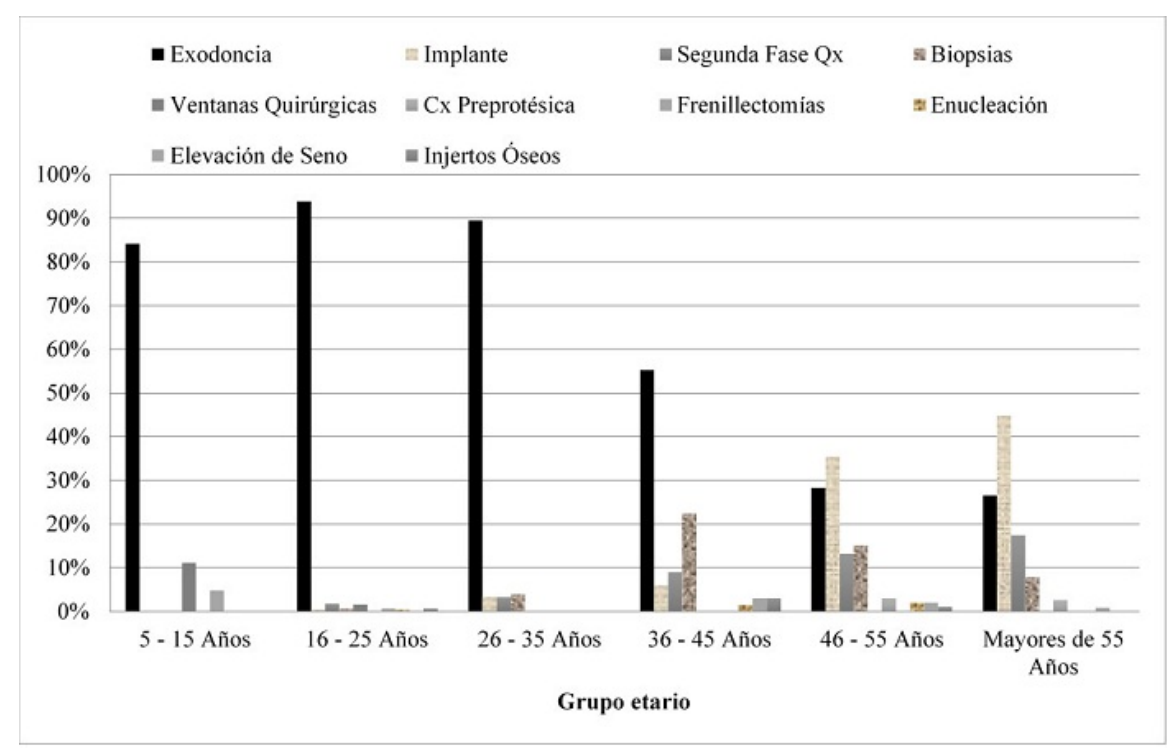

FIGURA 1

Distribución de los procedimientos según los grupos etarios del total de historias clínicas analizadas

$\mathrm{Al}$ analizar cada una de las historias clínicas, se encontraron 74 (7 \%) casos que durante el tratamiento presentaron algún tipo de situación desfavorable. El análisis del total de las historias estudiadas muestra que en los procedimientos de elevación de seno y de injertos óseos estuvo el mayor porcentaje de situaciones desfavorables (17\%) (tabla 2).

TABLA 2

Distribución de los eventos desfavorables de acuerdo con el número de procedimientos

\begin{tabular}{lcr}
\hline Procedimiento & Número de casos & $\begin{array}{c}\text { Situación } \\
\text { desfavorable } \\
(\mathbf{n} / \%)\end{array}$ \\
\hline Exodoncia & 738 & $66(9,0)$ \\
Implante & 149 & $3(2,0)$ \\
Biopsias & 57 & $2(3,5)$ \\
Ventanas quirúrgicas & 14 & $1(7,0)$ \\
Elevación de seno & 6 & $1(17,0)$ \\
Injertos óseos & 6 & $1(17,0)$ \\
Total & 1062 & $74(7,0)$ \\
\hline
\end{tabular}

A partir de las 74 historias con reporte de situación desfavorable se registraron las variables propuestas (tabla 3). El mayor número de situaciones (66) se encontró en exodoncia (89\%), dato que se esperaba, teniendo en cuenta que corresponde al procedimiento que se realiza en el área quirúrgica, tema de análisis de este estudio (tabla 3). Ello también explica que el grupo de 16-25 años fuera el más afectado por situaciones desfavorables, ya que es el grupo en el que se realiza el mayor número de exodoncias. La distribución de las variables demográficas fue similar a lo observado para al grupo total. El $57 \%$ correspondió a historias clínicas de pacientes del sexo femenino, y el 89 \% no presentaba afectación sistémica. 
TABLA 3

Distribución de las variables a partir de las historias clínicas con registro de situación desfavorable

\begin{tabular}{llr}
\hline Nombre de la variable & \multicolumn{1}{c}{$\begin{array}{c}\text { Número de } \\
\text { casos (n/\%) }\end{array}$} \\
\hline Procedimiento & Exodoncia & $66(89,0)$ \\
& Implante & $3(4,0)$ \\
& Biopsias & $2(2,7)$ \\
& Ventanas & $1(1,3)$ \\
& quirúrgicas & \\
& Elevación de seno & $1(1,3)$ \\
Grupos etarios & Injertos óseos & $1(1,3)$ \\
(en años) & $5-15$ & $5(7,0)$ \\
& $16-25$ & $29(39,0)$ \\
& $26-35$ & $17(23,0)$ \\
& $36-45$ & $7(9,0)$ \\
& $46-55$ & $2(3,0)$ \\
Tipo de & $>55$ & $14(19,0)$ \\
aseguramiento & Subsidiado & $12(16,2)$ \\
& Contributivo & $54(73,0)$ \\
& Vinculado & $6(8,1)$ \\
Estado civil & Ninguno & $292,7)$ \\
& Soltero(a) & $55(74,3)$ \\
& Casado(a) & $10(13,5)$ \\
& Unión libre & $4(5,4)$ \\
& Viudo(a) & $1(1,3)$ \\
& Divorciado(a) & $4(5,4)$ \\
\hline
\end{tabular}

Con base en la opinión de los cirujanos reunidos para el análisis por expertos, y teniendo en cuenta las definiciones conceptuales, las 74 situaciones desfavorables se tipificaron así: 41 complicaciones (55,4 \%), 22 indicios de atención insegura (29,7\%) y 11 eventos adversos (14,9\%). A su vez, los eventos adversos se clasificaron en prevenibles y no prevenibles y se encontró que solo dos casos fueron tipificados como no prevenibles (tabla 4).

Los eventos adversos se registraron solo durante procedimientos de exodoncia. La fractura de la tuberosidad se consideró un evento adverso que no es posible prevenir, a pesar de tomarse todas las medidas requeridas en este tipo de intervenciones. Los otros eventos adversos fueron considerados prevenibles. Referente a las complicaciones, la fractura radicular fue la que más se informó. Los indicios de atención insegura corresponden a inadecuado diligenciamiento de la historia clínica que deben corregirse para asegurar la calidad de la atención y evitar así posteriores eventos adversos durante el tratamiento (tabla 4). 
TABLA 4

Tipificación de las situaciones desfavorables registradas en historias clínicas de pacientes sometidos a procedimientos quirúrgicos

\begin{tabular}{|c|c|c|c|c|c|}
\hline Evento adverso & $\begin{array}{l}\text { Número } \\
\text { (\%) }\end{array}$ & Complicaciones & $\begin{array}{l}\text { Número } \\
(\%)\end{array}$ & $\begin{array}{l}\text { Atención } \\
\text { insegura }\end{array}$ & $\begin{array}{l}\text { Número } \\
(\%)\end{array}$ \\
\hline $\begin{array}{l}\text { Fractura de } \\
\text { tuberosidad** }\end{array}$ & $2(3)$ & $\begin{array}{l}\text { Fractura } \\
\text { radicular }\end{array}$ & $21(30)$ & $\begin{array}{l}\text { Evolución no } \\
\text { corresponde a } \\
\text { plan de } \\
\text { tratamiento }\end{array}$ & $12(16)$ \\
\hline $\begin{array}{l}\text { Fractura de } \\
\text { restauraciones }\end{array}$ & $2(3)$ & $\begin{array}{l}\text { Comunicación } \\
\text { oroantral }\end{array}$ & $5(7)$ & & \\
\hline adyacentes* & & Lesión nerviosa & $4(5)$ & & \\
\hline $\begin{array}{l}\text { Laceración de tejido } \\
\text { blando* }\end{array}$ & $2(3)$ & Alveolitis & $4(5)$ & $\begin{array}{l}\text { No hay } \\
\text { evolución }\end{array}$ & $8(11)$ \\
\hline $\begin{array}{l}\text { Luxación de diente } \\
\text { adyacente* }\end{array}$ & $1(1)$ & $\begin{array}{l}\text { Reacción a la } \\
\text { amoxicilina }\end{array}$ & $2(3)$ & & \\
\hline Deglución de diente* & $1(1)$ & $\begin{array}{l}\text { Pérdida de } \\
\text { implante }\end{array}$ & $2(3)$ & & \\
\hline $\begin{array}{l}\text { Desplazamiento a espacio } \\
\text { pterigomaxilar* }\end{array}$ & $1(1)$ & Hemorragia & $1(1)$ & $\begin{array}{l}\text { Consentimiento } \\
\text { no corresponde }\end{array}$ & $2(3)$ \\
\hline $\begin{array}{l}\text { Exposición de bola de } \\
\text { Bichat* }\end{array}$ & $1(1)$ & Hematoma & $1(1)$ & $\begin{array}{l}\text { a plan de } \\
\text { tratamiento }\end{array}$ & \\
\hline Crisis hipertensiva* & $1(1)$ & $\begin{array}{l}\text { Fracaso } \\
\text { injerto }\end{array}$ & $1(1)$ & & \\
\hline
\end{tabular}

\section{DISCUSIÓN}

Por la responsabilidad que compete en la atención en salud, es una obligación para las entidades prestadoras conocer los problemas que pueden surgir en las diferentes áreas clínicas y así establecer medidas de control y prevención. Por esta razón, la Facultad de Odontología de la Pontificia Universidad Javeriana ha venido trabajando en identificar los eventos adversos, complicaciones e indicios de atención insegura que le permitan asegurar la calidad de atención de sus pacientes. Es interesante resaltar que el evento adverso fue la situación que menos se presentó, resultado alentador, ya que puede ser un indicativo de que, a pesar de la complejidad de los procedimientos en cirugía oral, la atención es segura.

En la literatura sobre el tema son escasos los datos acerca de estas situaciones en la práctica clínica odontológica en general y, en particular, durante los tratamientos de cirugía oral, sumado al hecho de que ninguno es comparable, ya que no se realizan en instituciones que atienden pacientes bajo el modelo docenciaservicio. En este estudio se encontró que se presentaron eventos desfavorables durante la atención en el 7 \% de todos los procedimientos efectuados durante los años 2014 y 2015, en el área quirúrgica del Posgrado de Patología y Cirugía Oral de esta universidad. Entre estos eventos, el $14 \%$ se clasificó como eventos adversos, los cuales estuvieron en su totalidad asociados a exodoncias, principalmente de terceros molares.

Autores como Carvalho y colaboradores (18), Kandasamy y colaboradores (19) y Boloux y colaboradores (20) informaron que los eventos desfavorables más frecuentes ocurridos durante la exodoncia de terceros molares impactados son laceración de colgajo, fractura de la tuberosidad y daño al diente adyacente con grandes restauraciones o lesiones cariosas. Dichos hallazgos son similares a los de las historias analizadas en la presente investigación, entre las que los eventos adversos más frecuentes fueron: fractura d e la tuberosidad, 
laceración de tejidos blandos y fractura a dientes adyacentes. En el análisis de los eventos desfavorables se encontró un caso de desplazamiento del tercer molar al espacio pterigomaxilar $(1 \%$ de los eventos encontrados), situación que puede ocurrirle aun al cirujano más experimentado. Sin embargo, no hay que dejar de lado la correcta planificación prequirúrgica con el fin de evitar la ocurrencia de eventos graves como este (21).

Durante los procedimientos de cirugía oral, los pacientes con afectación sistémica, específicamente aquellos hipertensos sin un control adecuado de su condición, son susceptibles a desarrollar crisis hipertensivas ante el mínimo estímulo quirúrgico o episodio de estrés. Este tipo de evento se presentó en uno de los pacientes durante el procedimiento quirúrgico. Balasubramaniyan y colaboradores (22) establecieron que los procedimientos quirúrgicos que generan ansiedad en los pacientes inducen la liberación de catecolaminas endógenas que, junto con la administración de anestésicos locales con sustancias simpaticomiméticas, pueden elevar la presión arterial, la cual en casos graves también puede desencadenar incluso la muerte del paciente.

Los eventos adversos se clasificaron en dos tipos: no prevenibles (los cuales, a pesar de todas las medidas preventivas implementadas por el profesional y luego de haber analizado todos los factores de riesgo, es inevitable que ocurran) y prevenibles (que se presentan cuando el profesional no establece estrategias para evitar su ocurrencia y generan daño al paciente). Durante la revisión de atenciones relacionadas con eventos adversos, se encontró un caso de deglución de un diente supernumerario durante su exodoncia, evento suscitado en un paciente pediátrico no colaborador. Ante una posible situación como esta, es recomendable que los procedimientos se realicen bajo sedación o anestesia general. La exposición accidental de la bola de Bichat durante la extracción de un tercer molar superior se reportó en un caso, evento que está directamente relacionado con un abordaje amplio en exodoncias de terceros molares superiores. No se encontraron en la literatura casos que dieran cuenta de la frecuencia de este tipo de eventos.

Entre los eventos desfavorables clasificados como complicaciones se observó una mayor frecuencia de facturas radiculares durante la exodoncia. Nayyar y colaboradores (23) establecen que los molares y premolares maxilares son los dientes más propensos a fractura, debido a su morfología compleja. Por ello, debe realizarse un análisis riesgo-beneficio antes de intentar extraer una raíz fracturada, ya que se corre riesgo de desplazamiento a estructuras anatómicas circundantes, o su extracción puede dañar estructuras adyacentes.

La segunda complicación más frecuente encontrada en este estudio fue la comunicación oroantral en un porcentaje equivalente al 5,1\%, señalado por Del Rey-Santamaría y colaboradores (24). Este riesgo se presenta en todas las edades y depende de las variaciones anatómicas de cada paciente. Con respecto a las lesiones nerviosas, hubo una baja frecuencia. Se sabe que el riesgo de parestesia no es el mismo para todas las exodoncias, aunque se ha observado que la posición mesioangular (35-45 grados) presenta un mayor riesgo en una secuela por lesión nerviosa. Sin embargo, más del $95 \%$ de dientes incluidos no presentarán ninguna complicación posterior a su exodoncia (25).

A pesar de la poca frecuencia de lesiones en el nervio dentario inferior, se han desarrollado diferentes técnicas que se deben tener en cuenta en casos de alto riesgo con el fin de evitar su ocurrencia. Pogrel y colaboradores (26) plantean una técnica alternativa para aplicarse en pacientes que presentan íntima relación de las raíces del tercer molar al nervio dentario inferior, en los cuales realizar la exodoncia podría desencadenar una lesión nerviosa permanente. Consiste en efectuar un corte de la corona clínica con fresa quirúrgica dejando sumergidas las raíces $3 \mathrm{~mm}$, sin tratamiento endodóntico; el operador debe también proteger la cortical lingual en el momento de seccionar el diente, a fin de evitar daños al paquete vasculonervioso lingual (26). Además de la posición del tercer molar, Renton y colaboradores (27) indican que las mujeres presentan un mayor riesgo de lesiones nerviosas, debido a que acuden al odontólogo con mayor frecuencia que los hombres para realizarse los tratamientos. Esto coincide con los hallazgos de este estudio.

Con respecto a la osteítis alveolar que fue encontrada en el $5 \%$ de los casos, Bui y colaboradores (28) reportaron una frecuencia en un rango del $1 \%$ al $3 \%$ para las exodoncias en general. Esta complicación está 
relacionada con factores como la interacción entre paciente y profesional o condiciones propias del paciente que influyen en la formación del tapón plaquetario.

Las reacciones alérgicas a la amoxicilina se presentaron en un porcentaje del $3 \%$, siendo la erupción o salpullido (rash) cutáneo la manifestación más común. Se ha establecido que aproximadamente el $10 \%$ de los pacientes presenta reacciones a medicamentos betalactámicos, y del 0,1\% al 0,4\%, a la anafilaxia (27). Aunque en el actual estudio la reacción al antibiótico se consideró una complicación, ya que los pacientes desconocían esta sensibilidad, es importante resaltar que puede considerarse un evento adverso prevenible cuando el clínico no elabora una buena anamnesis. Adicionalmente, los pacientes pueden presentar una reacción alérgica a los betalactámicos después de una primera medicación (29).

En cuanto a la pérdida de implantes, existen diversos criterios sobre afecciones sistémicas que contraindican la colocación de un implante. Se incluyen la diabetes no controlada, trastornos sanguíneos, inmunosupresión, problemas cognitivos que interfieren en el cuidado de un implante y aplicación intravenosa de bifosfonatos. Estudios retrospectivos demuestran que factores sistémicos como hipertensión o afección coronaria no se han asociado al aumento de la tasa de fracaso temprano o tardío de implantes dentales y no existe evidencia que relacione la pérdida de un implante dental en pacientes diagnosticados con hipertensión arterial. Esto se relaciona con los hallazgos de este estudio, ya que se reportó la pérdida de un implante en el $3 \%$ de los pacientes, y uno de ellos tenía como antecedente hipertensión arterial (30).

Dada la frecuencia de eventos desfavorables relacionados con la exodoncia de terceros molares, surge el debate sobre las indicaciones para realizar este procedimiento con el ánimo de prevenir la aparición de complicaciones. Steed (31) indica que un tercer molar asintomático no refleja necesariamente la ausencia de enfermedad y debe considerarse la exodoncia de terceros molares cuando causan dolor, infección y patología ósea; cuando afectan los dientes adyacentes, o cuando intervengan con un tratamiento de ortodoncia planificado.

Además de lo anterior, Pratt y colaboradores (32) establecen otros criterios para realizar exodoncia de terceros molares: caries extensa con exposición pulpar, enfermedad periodontal, fracturas radiculares y desfocalización en pacientes con enfermedad cardiaca antes de una intervención quirúrgica. Friedman (25) reporta que solo el $12 \%$ de terceros molares impactados está asociado a condiciones patológicas como quistes o daños a dientes adyacentes. Las infecciones alrededor de los terceros molares ocurren en un porcentaje cercano al $10 \%$, las cuales pueden tratarse con antibióticos, enjuagues orales o remoción de excesos de tejido pericoronal, sin necesidad de realizar la exodoncia de dichos dientes. El profesional debe analizar estas condiciones durante la planeación del tratamiento, con el fin de evitar la ocurrencia de eventos desfavorables como los antes descritos (25).

Otras características como la posición y la profundidad del tercer molar en el hueso mandibular y la odontosección, que se realiza durante el acto quirúrgico, son factores que se relacionan directamente con la ocurrencia de eventos desfavorables, ya que el acceso quirúrgico puede llegar a ser tan amplio que las lesiones a estructuras anatómicas importantes como consecuencia del procedimiento pueden llegar a ser inevitables (33). Debido a la baja posibilidad de desarrollar condiciones patológicas en terceros molares incluidos, el operador debe desarrollar un criterio estricto en las indicaciones de la exodoncia de terceros molares con el objetivo de buscar el bienestar de su paciente y prevenir el desarrollo de un evento adverso.

$\mathrm{Al}$ analizar los indicios de atención insegura es obligatorio que el profesional sea cauteloso en la elaboración de la historia clínica y la descripción de los procedimientos, ya que una omisión de datos desencadenaría una atención insegura, una complicación o un evento adverso propiamente dicho. Es importante resaltar que cuando se presente algún tipo de estas situaciones, se registre, para contar con datos epidemiológicos reales como base para diseñar estrategias que prevengan los daños ocurridos por eventos adversos, para asegurar la atención de los pacientes y para evitar también inconvenientes legales. 


\section{CONCLUSIONES}

Los eventos desfavorables fueron escasos en los procedimientos realizados en el área quirúrgica, en su mayoría asociados a exodoncias. De igual manera, fueron poco frecuentes los eventos adversos y la mayoría de estos eran prevenibles.

\section{RECOMENDACIONES}

Completar los estudios sobre eventos adversos en la facultad, a efectos de implementar estrategias generales y específicas para cada una de las áreas de atención en salud oral. A partir de estos análisis, adicionar a los planes de estudios herramientas con las cuales profundizar en su abordaje y formas de prevención.

\section{Referencias}

Organización Mundial de la Salud (OMS). La alianza mundial para la seguridad del paciente. La investigación en seguridad del paciente. Mayor conocimiento para una atención más segura [internet]. Ginebra, Suiza: OMS; 2008. Disponible en: https://www.who.int/patientsafety/information_centre/documents/ps_research_broch ure_es.pdf

2. Villarreal Cantillo E. Seguridad de los pacientes: un compromiso de todos para un cuidado de calidad. Salud Uninorte. 2007 Mar; 23(1): 112-9.

3. Luengas S. Seguridad del paciente: un modelo organizacional para el control sistemático de los riesgos en la atención en salud [internet]. Bogotá, Colombia: Fundación Corona-Centro de Gestión Hospitalaria; 2009. Disponible en: https://es.calameo.com/read/0001476167d5dbd7ffa24

4. García-Barbero M. La alianza mundial para la seguridad del paciente [internet]. Valencia, España: Consejo de Enfermería de la Comunidad Valenciana; 2007. Disponible en: URL:https://www.portalcenova.es/es/grupos/ biologicos/pacientes/alianza_mundial_seguridad_paciente.pdf.

5. Ministerio de Salud y Protección Social, República de Colombia. Seguridad del paciente y la atención en salud. Guía técnica "Buenas prácticas para la seguridad del paciente en la atención en salud" [internet]. Bogotá, Colombia: el Ministerio; 2015. Disponible en: https://www.minsalud.gov.co/sites/rid/Lists/BibliotecaDigital/RIDE/DE /CA/Guia-buenas-practicas-seguridad-paciente.pdf.

6. Ministerio de la Protección Social, República de Colombia. Lineamientos para la implementación de la Política de Seguridad el Paciente [internet]. Bogotá, Colombia: el Ministerio; 2008. Disponible en: https://minsalud.gov. co/Normatividad_Nuevo/Forms/Top\%20Normatividad.aspx.

7. Ministerio de la Protección Social, República de Colombia. Herramientas para promover la estrategia de la seguridad del paciente en el Sistema Obligatorio de Garantía de la Calidad de la Atención en Salud [internet]. Bogotá, Colombia: el Ministerio; 2008.

8. Tafur MC, Camacho LD, Mejía SH, González J, Huertas MF. Frecuencia de eventos adversos de la terapia endodóntica y seguimiento de pacientes atendidos en el Posgrado de Endodoncia de la Pontificia Universidad Javeriana (2007-2008). Univ Odontol. 2014 jul-dic; 33(71). https://dx.doi.org/doi:1011144/Jjaveriana.uo3371.feat.

9. Tafur DJ, Ramírez GP, Cárdenas CA, Serrano JJ, Sarralde AL, Camacho SP, Rodríguez A, González J. Características y prevalencia de los eventos adversos presentados en la Clínica del Posgrado de Periodoncia de la Facultad de Odontología de la Pontificia Universidad Javeriana durante el período 2011-2012. Univ Odontol. 2016 jul-dic; 35(75). https://dx.doi.org/10.11144/Javeriana.uo35-75.cpea

10. Huertas MF, González J, Camacho S, Sarralde AL, Rodríguez A. Analysis of the adverse events reported to the office of the clinical director at a dental school in Bogotá, Colombia. Acta Odontol Latinoam. 2017 Apr; 30(1): 19-25. 
11. Morejón Álvarez F, López Benítez H, Morejón Álvarez T, Corbo Rodríguez MT. Presentación de un estudio en 680 pacientes operados de terceros molares retenidos. Rev Cubana Estomatol. 2000 may-ago; 37(2): 102-5.

12. García-Peñín A, Guisado-Moya B, Montalvo-Moreno JJ. Riesgos y complicaciones de la anestesia local en la consulta dental. Estado actual. RCOE. 2003 ene-feb; 8(1): 41-63.

13. Liu W, Yang X, Li C, Mo A. Adverse drug reactions to local anesthetics: A systematic review. Oral Surg Oral Med Oral Pathol Oral Radiol. 2013 Mar; 115(3): 319-27. https://doi.org/10.1016/j.oooo.2012.04.024

14. Aboul-Hosn S, Sieira Gil R, Monner Diéguez A. Una rara complicación en la extracción del tercer molar inferior incluido: Caso clínico. Rev Esp Cir Oral Maxilofac. 2009 sep-oct; 31(5): 333-6.

15. Piccinni C, Gissi DB, Gabusi A, Montebugnoli L, Poluzzi E. Paraesthesia after local anaesthetics: An analysis of reports to the FDA adverse event reporting system. Basic Clinic Pharmacol Toxicol. 2015 Jul; 117(1): 52-6. ht tps://doi.org/10.1111/bcpt.12357

16. Perea-Pérez B, Labajo-González E, Santiago-Sáez A, Albarrán-Juan E, Villa-Vigil A. Analysis of 415 adverse events in dental practice in Spain from 2000 to 2010. Med Oral Patol Oral Cir Bucal. 2014 sep; 19(5): e500-5. https ://doi.org/10.4317/medoral.19601

17. Sebastiani AM, Barancelli Todero SR, Gabardo G, da Costa DJ, Barbosa Rebelatto NL, Scariot R. Intraoperative accidents associated with surgical removal of third molars. Braz J Oral Sci. 2014 Oct-Dec; 13(4): 276-80.

18. Carvalho R, Araújo-Filho R, Vasconcelos B. Adverse events during the removal of impacted maxillary third molars. Int J Oral Maxillofac Surg. 2014 Sep; 43(9): 1142-7. https://doi.org/10.1016/j.ijom.2014.04.002

19. Kandasamy S, Rinchuse DJ, Rinchuse DJ. The wisdom behind third molar extractions. Aust Dent J. 2009 Dec; 54(4): 284-92. https://doi.org/10.1111/j.1834-7819.2009.01152.x

20. Bouloux GF, Steed MB, Perciaccante VJ. Complications of third molar surgery. Oral Maxillofac Surg Clin North Am. 2007 Feb; 19(1): 117-28.

21. Liceaga Escalera CJ, Castañeda Sánchez O, González Olmedo VM. Extracción de tercer molar superior desplazado a espacio pterigomaxilar apoyada por fluoroscopio. Rev ADM. 2013 feb; 70(6): 324-8.

22. Balasubramaniyan N, Rayapati DK, Puttiah RH, Tavane P, Singh SE, Rangan V, Kalakunta PR. Evaluation of anxiety induced cardiovascular response in known hypertensive patients undergoing exodontia. A prospective study. J Clin Diagn Res. 2016 Aug; 10(8): ZC123-7. https://doi.org/10.7860/JCDR/2016/19685.8391

23. Nayyar J, Clarke M, O'Sullivan M, Stassen LF. Fractured root tips during dental extractions and retained root fragments. A clinical dilemma? Br Dent J. 2015 Mar; 218(5): 285-90. https://doi.org/10.1038/sj.bdj.2015.147

24. Del Rey-Santamaría M, Valmaseda Castellón E, Berini Aytés L, Gay Escoda C. Incidence of oral sinus communications in 389 upper third molar extraction. Med Oral Patol Oral Cir Bucal, 2006 Jul; 11(4): E334-8.

25. Friedman JW. The Prophylactic extraction of third molars: a public health hazard. Am J Public Health. 2007 Sep; 97(9): 1554-9.

26. Pogrel MA, Lee JS, Muff DF. Coronectomy: a technique to protect the inferior alveolar nerve. J Oral Maxillofac Surg. 2004 Dec; 62(12): 1447-52.

27. Renton T, Yilmaz Z, Gaballah K. Evaluation of trigeminal nerve injuries in relation to third molar surgery in a prospective patient cohort. Recommendations for prevention. Int J Oral Maxillofac Surg, 2012 Dec; 41(12): 1509-18. https://doi.org/10.1016/j.ijom.2012.06.025

28. Bui CH, Seldin EB, Dodson TB. Types, frequencies, and risk factors for complications after third molar extraction. J Oral Maxillofac Surg. 2003 Dec; 61(12): 1379-89.

29. Chang C, Mahmood MM, Teuber SS, Gershwin ME. Overview of penicillin allergy. Clin Rev Allergy Immunol. 2012 Aug; 43(1-2): 84-97. https://doi.org/10.1007/s12016-011-8279-6

30. Domínguez J, Acuña J, Rojas M, Bahamondes J, Matus S. Estudio de asociación entre enfermedades sistémicas y el fracaso de implantes dentales. Rev Clin Periodoncia Implantol Rehabil Oral. 2013 abr; 6(1): 9-13. https://d oi.org/10.4067/S0719-01072013000100002

31. Steed MB. The indications for third-molar extractions. J Am Dent Assoc. 2014 Jun; 145(6): 570-3. https://doi. org/10.14219/jada.2014.18 
32. Pratt CA, Hekmat M, Barnard JD, Zaki GA. Indications for third molar surgery. J R Coll Surg Edinb. 1998 Apr; 43(2): 105-8.

33. Flores Ramos JM, Ochoa Zaragoza MG, Barraza Salas JH, Romero Paredes JJ, Rojas García MC. Complicaciones postoperatorias asociadas a la cirugía del tercer molar inferior retenido. Rev ADM. 2015 sep; 72(6): 314-9.

Licencia Creative Commons CC BY 4.0

Cómo citar: Pesántez Alvarado JM, Camacho Ladino JD, Rodríguez Ciódaro A, Camacho Peña SP, Sarralde Delgado AL, Castro Haiek DE, González Moncada J. Análisis de los eventos desfavorables como resultado de la atención en cirugía oral. Univ Odontol. 2017 jul-dic; 36(77). https://doi.org/10.11144/Jav eriana.uo36-77.aeco 